\title{
Prostate-Specific Membrane Antigen PET/ Magnetic Resonance Imaging for the Planning of Salvage Radiotherapy in Patients with Prostate Cancer with Biochemical Recurrence After Radical Prostatectomy
}

Mattijs Elschot, $\mathrm{PhD}^{\mathrm{a}, \mathrm{b}, *}$, Kirsten Margrete Selnæs, $\mathrm{PhD}^{\mathrm{b}}$,

Sverre Langørgen, $M D^{b}$, Håkon Johansen, $M D^{b}$,

Helena Bertilsson, MD, $\mathrm{PhD}^{\mathrm{c}, \mathrm{d}}$, Torgrim Tandstad, $\mathrm{MD}, \mathrm{PhD}^{\mathrm{c}, \mathrm{e}}$,

Tone Frost Bathen, $\mathrm{PhD}^{\mathrm{a}, \mathrm{f}}$

\section{KEYWORDS}

- Prostate cancer $\bullet$ Recurrence $\bullet$ Prostatectomy $\bullet$ PSMA $\bullet$ PET $\bullet$ MR imaging

- Salvage radiotherapy

\section{KEY POINTS}

- Prostate-specific membrane antigen (PSMA) PET/magnetic resonance (MR) imaging can help distinguish between patients with prostate cancer with locoregional recurrence and those with distant metastases, even at low prostate-specific antigen levels.

- PSMA PET/MR imaging may have advantages compared with PET/computed tomography for the detection of local recurrence and anatomic correlates to PET-positive lymph node and bone lesions.

- PSMA PET/MR imaging can help in making informed treatment decisions in patients with biochemical recurrence after radical prostatectomy.

- PSMA PET/MR imaging enables dose-escalated and metastases-directed salvage radiotherapy in patients with biochemical recurrence after radical prostatectomy.

Disclosures: The researcher position of M. Elschot at the Norwegian University of Science and Technology is funded by the Central Norway Regional Health Authority. K.M. Selnæs, S. Langørgen, H. Johansen, H. Bertilsson, T. Tandstad, and T.F Bathen have nothing to disclose.

a Department of Circulation and Medical Imaging, Norwegian University of Science and Technology, PO Box 8900, NO-7491 Trondheim, Norway; ${ }^{b}$ Department of Radiology and Nuclear Medicine, St. Olavs Hospital, Trondheim University Hospital, PO Box 3250 Torgarden, NO-7006 Trondheim, Norway; ' Department of Cancer Research and Molecular Medicine, Norwegian University of Science and Technology, PO Box 8900, NO-7491 Trondheim, Norway; d Department of Urology, St. Olavs Hospital, Trondheim University Hospital, PO Box

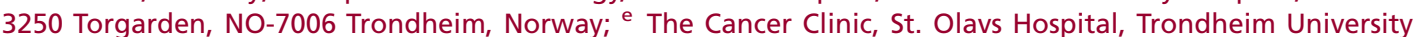
Hospital, PO Box 3250 Torgarden, NO-7006 Trondheim, Norway; ${ }^{f}$ Department of Circulation and Medical Imaging. Norwegian University of Science and Technology, PO Box 8900, NO-7491 Trondheim, Norway

* Corresponding author. Department of Circulation and Medical Imaging, Norwegian University of Science and Technology, PO Box 8900, 7491 Trondheim, Norway.

E-mail address: mattijs.elschot@ntnu.no 


\section{INTRODUCTION}

Prostate cancer is the second most commonly diagnosed cancer type among men worldwide. ${ }^{1}$ Over the last decades, the number of patients increased drastically because of increasing incidence and the wide availability of serum prostate-specific antigen (PSA) tests. Prostate cancer is a highly heterogeneous disease and patients are stratified in risk groups based on clinical factors such as PSA level, T stage, and Gleason grade. ${ }^{2}$ Patients in the low-risk group usually do not require immediate treatment, but can be followed on active surveillance until disease progression warrants intervention. In contrast, patients in the intermediate-risk or high-risk group are usually offered radical treatment. Radical prostatectomy (RP) and radiotherapy (RT) are the 2 most common options for treatment with curative intent, with the former currently most used. However, approximately $30 \%$ of patients experience disease recurrence within 5 years after surgical removal of the prostate. . $^{3,4}$

Suspicion of prostate cancer recurrence is based on changes in PSA kinetics during the follow-up after initial treatment. After RP, biochemical recurrence (BCR) is defined as 2 consecutive increasing PSA values greater than $0.2 \mathrm{ng} / \mathrm{mL} .{ }^{5}$ To decide on treatment of recurrent disease, it is of utmost importance to distinguish between patients who have locoregional confined disease, which is considered curable, and those with distant metastases. Salvage radiotherapy (sRT) to the prostate bed and possibly pelvic lymph nodes, with or without short-term androgen deprivation therapy (ADT), is the standard treatment option for the first group, whereas palliative long-term ADT is the common treatment option for the latter. ${ }^{5}$ However, patient stratification and treatment planning based on PSA values alone are challenging, because visual confirmation of the site of recurrence is lacking. Conventional imaging modalities are not sensitive enough to detect recurrent prostate cancer at the low PSA values with which patients typically present at the hospital. ${ }^{5}$ Importantly, this caveat leads to a more or less blind treatment approach with associated risks of overtreatment of patients with occult metastatic disease, and suboptimal treatment of patients with potentially curable disease. An imaging examination that provides better knowledge of the site of recurrence would alleviate these problems and could enable more personalized treatment strategies.

Simultaneous PET/magnetic resonance (MR) imaging shows potential for improving the diagnosis in several cancer types. ${ }^{6,7}$ For prostate cancer,
PET/MR imaging with prostate-specific membrane antigen (PSMA) ligands can provide images with excellent soft tissue contrast, as well as a superior sensitivity for detection of recurrent disease. ${ }^{8}$ This article discusses the emerging role of PSMA PET/ $M R$ imaging for the planning of SRT in patients with prostate cancer with BCR after RP.

\section{SALVAGE RADIOTHERAPY AFTER RADICAL PROSTATECTOMY}

$\mathrm{sRT}$ is defined as the administration of RT to the prostatic bed and possibly to the surrounding tissues, including lymph nodes, in patients with BCR after initial RP but no evidence of distant metastatic disease. ${ }^{9}$ The main advantage of sRT compared with adjuvant $\mathrm{RT}(\mathrm{aRT})$, which is the administration of RT after RP but before evidence of disease recurrence, is that the former avoids overtreatment and associated side effects in patients who would never develop recurrent cancer. ${ }^{9}$ One retrospective study with 510 patients found that the 8-year metastasisfree survival and overall survival did not differ significantly between pT3NO patients receiving aRT (92\% and $89 \%$, respectively) or observation plus early SRT $\left(91 \%\right.$ and $92 \%$, respectively). ${ }^{10}$ Three ongoing prospective randomized controlled trials (ClinicalTrials.gov identifiers: NCT00541047, NCT00860652, NCT00667069) hope to shed more light on the potential differences in cancer control between aRT and sRT. Convincing evidence shows that SRT is most effective at low PSA values and should be commenced at the first signs of recurrence. ${ }^{11-13}$ In a large multicenter study with patients initially treated with RP, Tendulkar and colleagues ${ }^{13}$ reported 5 -year absence of BCR in $71 \%$ of patients with pre-sRT PSA levels between 0.01 and $0.2 \mathrm{ng} /$ $\mathrm{mL}, 63 \%$ for PSA 0.21 to $0.5 \mathrm{ng} / \mathrm{mL}, 54 \%$ for 0.51 to $1 \mathrm{ng} / \mathrm{mL}, 43 \%$ for 1.01 to $2 \mathrm{ng} / \mathrm{mL}$, and $37 \%$ for PSA greater than $2 \mathrm{ng} / \mathrm{mL}$. Likewise, the cumulative rate of distant metastases significantly correlated with pre-sRT PSA levels, ranging from $9 \%$ to $27 \%$ for the lowest and highest PSA groups, respectively. Results of the Radiation Therapy Oncology Group (RTOG) 9601 randomized controlled trial showed that the addition of 24 months of ADT to early sRT resulted in significantly higher rates for overall survival $(76.3 \%$ vs $71.3 \%$ ) and lower incidences of metastatic disease (14.5\% vs $23 \%)$ and death from prostate cancer (5.8\% vs $13.4 \%$ ) at 12 years. ${ }^{14}$ In the GETUGAFU 16 randomized clinical trial, the addition of short-term ADT to early SRT had a significantly favorable effect on biochemical or clinical progression at 5 -year follow-up ( $80 \%$ for sRT + ADT vs $62 \%$ for sRT alone), but no benefit on overall survival was found at time of analysis. ${ }^{15}$ Similar results 
were recently reported from the interim analysis of the RTOG 0534 SPPORT trial, with 5-year freedom from progression in $71.1 \%$ of the patients who received prostate bed SRT alone versus $82.7 \%$ of patients who received sRT plus short-term ADT. A third arm with patients receiving additional irradiation of the pelvic lymph nodes showed significantly increased freedom from progression (89.1\%) compared with the other 2 arms, as well as a significantly reduced likelihood of developing distant metastases at 8-year follow-up. ${ }^{16}$ The reduced likelihood of developing distant metastases represents the first evidence from a randomized controlled trial that extending the sRT field to the pelvic lymph nodes leads to clinically meaningful reductions in $\mathrm{BCR}$ in patients without previous evidence of lymph node involvement (NO/Nx).

Computed tomography (CT) and bone scintigraphy (BS) are conventionally used to detect evidence of distant metastatic disease. However, these imaging modalities rarely detect disease at PSA levels less than $10 \mathrm{ng} / \mathrm{mL}$, which limits their use for most candidates for sRT. ${ }^{5}$ Consequently, patient selection for $\mathrm{SRT}$ and consecutive treatment planning is often based on PSA kinetics and other clinical parameters alone, without visual evidence of the site of recurrence. In general, a patient with prostate cancer with BCR after RP is considered a candidate for SRT if benefit from treatment may be expected, taking into consideration the risk of death unrelated to prostate cancer. The expected benefit of SRT is usually calculated using nomograms that typically include clinical parameters such as pre-sRT PSA, PSA doubling time, Gleason score, seminal vesicle invasion, extracapsular extension, surgical margins, lymph node metastases, concurrent or neoadjuvant ADT, and radiation dose to predict the risk of being progression free at some time after treatment. ${ }^{11,13}$ In general, men with a life expectancy greater than 10 years may benefit from sRT, often given in combination with short-term ADT or antiandrogens (6-24 months). The total radiation dose given to the prostate bed should be at least 66 Gy. ${ }^{5}$ A total radiation dose of 45 Gy to the whole pelvis is usually given in relapsing patients who had positive nodes on pelvic lymph node dissection (N1), although recent evidence shows that patients with negative or unknown pelvic lymph node stage (NO/Nx) may also benefit from pelvic lymph node irradiation. ${ }^{16}$

\section{PROSTATE-SPECIFIC MEMBRANE ANTIGEN PET IMAGING}

Clinical parameters are useful for predicting the patient's population-based probability of benefiting from sRT. ${ }^{11,13}$ However, they do not provide direct evidence of the location of recurrence, which is of paramount importance for more tailored treatment. The ideal imaging examination would detect local recurrence in the prostate bed $(T+)$, lymph node metastases inside (N1) and outside the pelvis (M1a), bone metastases (M1b) and other distant metastases (M1c) at low PSA levels, in order to facilitate informed clinical decision making based on the tumor-node-metastasis (TNM) staging system. However, traditional imaging modalities all have their limitations; CT is not useful for detection of local recurrence because of lack of soft tissue contrast, whereas both CT and BS lack sensitivity for detection of lymph node and bone metastases at low PSA levels. ${ }^{17}$ MR imaging is excellent for assessment of local recurrence ${ }^{18}$ but also performs poorly for detection of small lymph node metastases. ${ }^{19}$

Lately, there has been a strong focus on the development of prostate cancer-specific PET radiotracers, because alterations in molecular processes typically precede morphologic changes, thus providing opportunities for early detection of the site of recurrence. ${ }^{11} \mathrm{C}$-choline and ${ }^{18} \mathrm{~F}$-fluciclovine are radiotracers that are currently US Food and Drug Administration approved for PET imaging in recurrent prostate cancer, whereas ${ }^{18} \mathrm{~F}$-fluoromethylcholine is often used in clinical practice as well. Most recently, PSMA-based radiotracers that are typically labeled with ${ }^{68} \mathrm{Ga}$ or ${ }^{18} \mathrm{~F}$, depending on the subtype, are rapidly gaining popularity for detection of recurrent prostate cancer. The current European Association of Urology (EAU) guidelines state that choline PET/CT may be useful in selected patients with PSA level greater than $1 \mathrm{ng} / \mathrm{mL}$, especially when PSA doubling time is less than 6 months. ${ }^{5}$ However, it has been consistently shown that choline PET has low detection rates at PSA levels less than $2 \mathrm{ng} / \mathrm{mL} .{ }^{20-22}$ In a head-to-head comparison in 100 patients with BCR after RP, fluciclovine PET showed higher detection rates than choline PET at PSA levels less than $2 \mathrm{ng} / \mathrm{mL}(21 \%$ vs $14 \%$ for $\mathrm{PSA}<1 \mathrm{ng} / \mathrm{mL}, 45 \%$ vs $29 \%$ for PSA 1-2 $\mathrm{ng} / \mathrm{mL}){ }^{23}$ Several studies compared PSMA PET with choline PET scans of the same patients, and higher detection rates were consistently found for the former, especially at PSA levels less than $1 \mathrm{ng} / \mathrm{mL} .{ }^{24-26}$ In a recent review of the available literature on these 3 radiotracers, Evans and colleagues ${ }^{27}$ reported median PSMA PET detection rates of $51.5 \%$ for PSA level less than $1 \mathrm{ng} / \mathrm{mL}, 74 \%$ for PSA 1 to $2 \mathrm{ng} / \mathrm{mL}$, and $90.5 \%$ for PSA greater than $2 \mathrm{ng} / \mathrm{mL}$. These values compared favorably with those of choline PET $(19.5 \%$ for PSA level $<1 \mathrm{ng} / \mathrm{mL}, 44.5 \%$ for 
PSA $1-2 \mathrm{ng} / \mathrm{mL}$, and $76 \%$ for PSA $>2 \mathrm{ng} / \mathrm{mL}$ ) and fluciclovine PET $(38 \%$ for PSA $<1 \mathrm{ng} / \mathrm{mL}$, $65 \%$ for PSA $1-2 \mathrm{ng} / \mathrm{mL}$, and $78 \%$ for PSA $>2 \mathrm{ng} / \mathrm{mL}$ ). A large meta-analysis by Perera and colleagues ${ }^{28}$ reported similar pooled detection rates for PSMA PET at low PSA levels. Specifically for patients with $B C R$ after RP, these were $33 \%$ for PSA 0 to less than $0.2 \mathrm{ng} / \mathrm{mL}$, $46 \%$ for PSA 0.2 to less than $0.5 \mathrm{ng} / \mathrm{mL}, 57 \%$ for PSA 0.5 to less than $1 \mathrm{ng} / \mathrm{mL}, 82 \%$ for PSA 1 to less than $2 \mathrm{ng} / \mathrm{mL}$, and $97 \%$ for PSA greater than $2 \mathrm{ng} / \mathrm{mL}$. Stratified by the site of recurrence, the pooled estimate of PSMA PET positivity for this patient group was $22 \%$ in the prostate bed, $36 \%$ in the pelvic lymph nodes, $7 \%$ in the extrapelvic lymph nodes, $15 \%$ in the bones, and $2 \%$ in the distant viscera.

In summary, PSMA ligands seem to outperform other prostate cancer-specific PET tracers, especially because of their ability to detect recurrent disease at PSA levels less than $1 \mathrm{ng} / \mathrm{mL}$. Recurrence is more often detected outside than inside the prostate bed, ${ }^{28}$ even at PSA levels less than $0.5 \mathrm{ng} / \mathrm{mL},{ }^{29}$ which indicates that offering SRT to the prostate bed alone is suboptimal for most patients. One limitation is that reliable histologic verification of the PSMA PET findings is often lacking in the setting of recurrent prostate cancer. Although some studies have used biopsies to confirm subsets of PSMA PET-positive lesions, this approach does not provide information on lesions that are false-negative on imaging. Consequently, the best estimates of the sensitivity and specificity of PSMA PET in patients with BCR after $\mathrm{RP}$ are obtained from patients who have undergone dissection of the pelvic lymph nodes for primary staging. The pooled sensitivity and specificity of PSMA PET in this setting were shown to be $77 \%$ and $97 \%$, respectively, on a per-patient analysis, and $75 \%$ and $99 \%$, respectively, on a per-lesion analysis. ${ }^{30}$

\section{PROSTATE-SPECIFIC MEMBRANE ANTIGEN PET/COMPUTED TOMOGRAPHY VERSUS PET/ MAGNETIC RESONANCE IMAGING}

Modern PET scanners are combined with a CT or MR imaging scanner to provide anatomic reference and morphologic correlates that are complementary to the molecular information from PET. To date, most PSMA PET studies have been performed on PET/CT scanners and some on PET/ MR imaging scanners, reflecting the different availability of these scanners. Nevertheless, there may be advantages of using PET/MR imaging instead of PET/CT for detection of prostate cancer recurrence, which can primarily be attributed to the superior soft tissue contrast of MR imaging compared with CT, as described later.

After RP and lymph node dissection, the anatomy of the pelvis is greatly changed. Multiparametric MR imaging (mpMR imaging), which is the combination of anatomic T2-weighted (T2w) MR images and functional dynamic contrastenhanced (DCE) and diffusion-weighted (DW) MR images, allows differentiation between recurrent cancer, residual prostate tissue, inflammatory tissue, and fibrosis. ${ }^{18}$ Although T2w MR imaging is mainly used for anatomic reference, the addition of DCE and, to some extent, DW MR imaging has been shown to significantly increase the sensitivity to detect locally recurrent disease. ${ }^{31}$ Although the complementary nature of PSMA PET and MR imaging for detection of local recurrence remains to be clarified in large prospective studies, initial work with limited patient numbers and varying MR imaging protocols generally shows a favorable effect of combining the two modalities. In their study with 119 relapsing patients who underwent PSMA PET/CT and subsequent $\mathrm{PET} / \mathrm{MR}$ imaging, Freitag and colleagues ${ }^{32}$ found that mpMR imaging detected 18 cases of local recurrence, of which 9 were missed by the PET components of both PET/CT and PET/MR imaging. Lutje and colleagues ${ }^{33}$ also detected more local recurrences with PET/MR imaging than with PET/CT (14 vs 9 in 25 patients), which was mainly attributed to the availability of MR images. In a small study with a trimodal PET/CT-MR imaging system, the addition of MR imaging was useful for cases with local recurrence, classified as indeterminate on PSMA PET/CT. ${ }^{34}$ Lake and colleagues $^{35}$ found that DCE MR imaging was most effective for identifying PSMA-avid foci in the prostatic bed, and was able to detect additional lesions without associated PSMA uptake. The potential of PSMA PET/MR imaging for detection of local recurrence is further shown in Fig. 1, in which the mpMR imaging provides morphologic and functional correlates for a region with suspicious focal PSMA uptake in the prostate bed.

For detection of lymph node and bone metastases, superiority of PSMA PET compared with both MR imaging and CT has been clearly shown. ${ }^{36-38}$ Nevertheless, PET/MR imaging may also have advantages compared with $\mathrm{PET} / \mathrm{CT}$ in this setting. Freitag and colleagues ${ }^{38}$ found that the visibility of the lymph nodes was significantly higher on MR imaging from PET/MR imaging compared with low-dose CT from PET/CT, as was the overall conspicuity for bone lesions. Similarly, AfsharOromieh and colleagues ${ }^{39}$ stated that the different sequences and the higher resolution of MR 
A

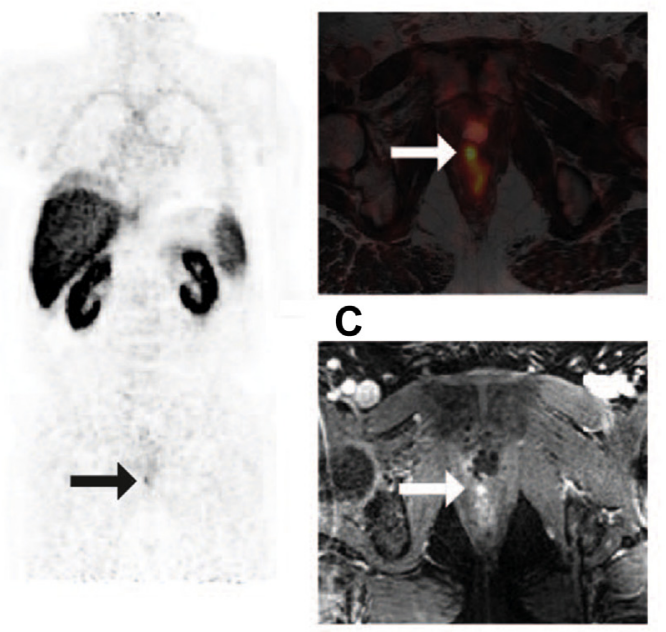

D

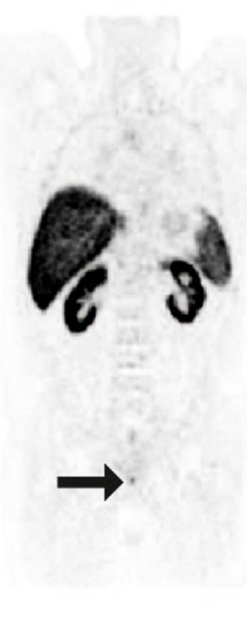

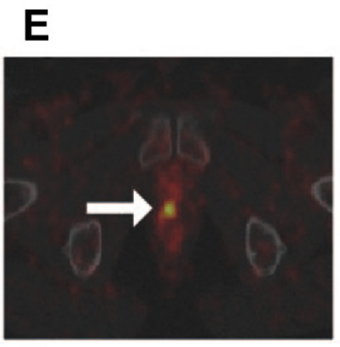

$\mathbf{F}$

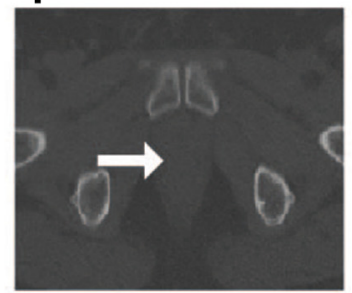

Fig. 1. PSMA-1007 PET/MR $(A-C)$ and PET/CT $(D-F)$ images of a patient with suspected local recurrence (arrows). Both PET from PET/MR imaging $(A)$ and PET from PET/CT $(D)$ show focal uptake in the prostate bed on the coronal images. PET image fusion with transverse T2w SPACE image provides soft tissue contrast for anatomic reference $(B)$, whereas PET image fusion with the transverse low-dose CT image does not $(E)$. Furthermore, early enhancement on the transverse DCE image provides another functional correlate $(C)$, which is lacking on the low-dose $C T$ image $(F)$.

imaging enabled a subjectively easier evaluation of the PET/MR images compared with the PET/CT images. Lake and colleagues $^{35}$ reported that anatomic correlates were found on MR imaging for all suspected lymph node and bone metastases with focal PSMA PET uptake. These findings are supported by Figs. 2 and 3 . They show that morphologic correlates to lymph node and bone lesions with focal PSMA uptake can be more easily identified on MR imaging than on low-dose CT.

The PET components of PET/CT and PET/MR imaging systems generally provide images of similar diagnostic quality, although standardized uptake values may differ because of differences in detector type and attenuation and scatter correction techniques. ${ }^{40}$ Ringheim and colleagues ${ }^{40}$ randomized the same-day scan order of PSMA PET/MR imaging and PET/CT and found that all suspected sites of local recurrence, lymph node, and bone metastases were visualized by both PET modalities. Similarly, Freitag and colleagues $^{38}$ found very high concordance for the detection of suspicious lymph nodes (98.5\%) and bone metastases (100\%) between PET from $\mathrm{PET} / \mathrm{MR}$ imaging and PET from PET/CT. Of special concern are the photopenic (halo) artifacts around the bladder and kidneys that are sometimes present in PET images from PET/MR imaging but not from PET/CT. ${ }^{38,39}$ These artifacts may especially hamper the evaluation of local recurrence and lymph node metastases in ${ }^{68} \mathrm{Ga}-\mathrm{PSMA}-11$ and ${ }^{18} \mathrm{~F}$-DCFPyl PET images, because these
PSMA variants are predominantly cleared by the kidneys. Halo artifacts are of less concern for ${ }^{18} \mathrm{~F}-\mathrm{PSMA}-1007$, which has a predominant hepatic clearance. ${ }^{41}$ Furthermore, it has been shown that, with simple adjustment of the maximum scatter fraction, these artifacts can be resolved. ${ }^{42}$ One other concern with regard to PET/MR imaging is the long scan time, which may reduce its cost-effectiveness. Scan protocols may vary between PET/MR imaging scanners and PSMA ligands, ${ }^{32,35,43}$ and international guidelines do not yet exist. In our institution, the total scan time of a whole-body ${ }^{18} \mathrm{~F}-\mathrm{PMSA}-1007$ PET/MR imaging examination (4 bed positions, thighs to chin), including mpMR imaging of the prostate bed, is less than 45 minutes (Table 1). In clinical practice, this means that with PET/MR imaging 1 patient can be scanned every hour, whereas 2 patients per hour can be examined with PET/CT. In case of the latter, separate mpMR imaging of the prostate bed is also performed, usually in combination with MR imaging of the spine and the pelvic and abdominal lymph nodes, unless recent MR images are available. Consequently, the total scan time for PSMA PET/MR imaging is shorter than that of PMSA PET/CT plus MR imaging, with the additional advantage of intrinsically coregistered images.

The incorporation of MR imaging in RT target volume delineation has been shown to decrease the interobserver contouring variability compared with CT alone in patients with primary and 
A

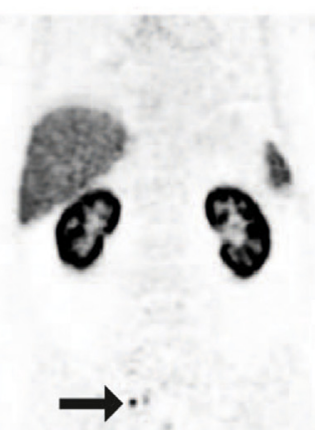

B

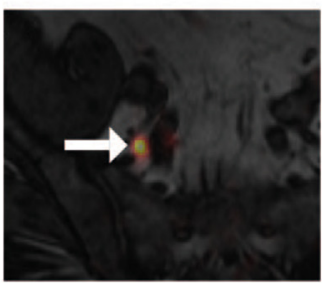

C

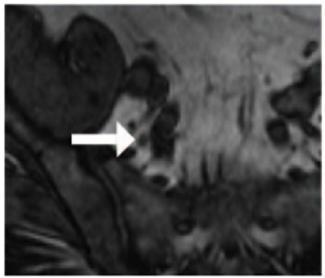

D

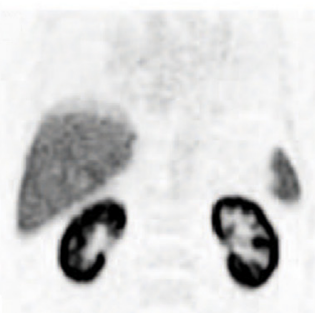

E
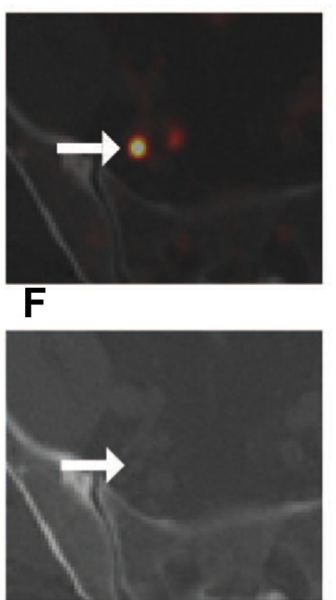

Fig. 2. PSMA-1007 PET/MR $(A-C)$ and PET/CT $(D-F)$ images of a patient with suspected lymph node metastases (arrows). Both PET from PET/MR imaging $(A)$ and PET from PET/CT $(D)$ show focal uptake in lymph nodes on the coronal images. PET image fusion with the transverse T1 weighted (T1w) volumetric interpolated breathhold examination (VIBE) Dixon image provides soft tissue contrast for anatomic reference $(B)$, whereas PET image fusion with the transverse low-dose $C T$ image does not $(E)$. Morphologic correlates to the suspected lymph nodes are more easily detected on the transverse T1w VIBE Dixon image $(C)$ than on the transverse low-dose CT image $(F)$.

recurrent prostate cancer. ${ }^{44,45}$ However, MR imaging does not intrinsically provide information on the tissue's electron density for dose calculation and may suffer from geometric distortions, which traditionally limited its role as a standalone modality for RT planning. Now modern MR imaging hardware and software solutions have largely resolved these issues, MR imaging-only workflows are being implemented for RT of primary prostate cancer in clinical practice. ${ }^{46}$ However, an MR imaging-only work flow would not be sufficient for dose-escalated and metastases-directed SRT in patients with prostate cancer with BCR after RP. Some investigators
A

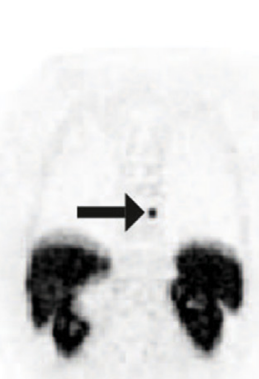

B

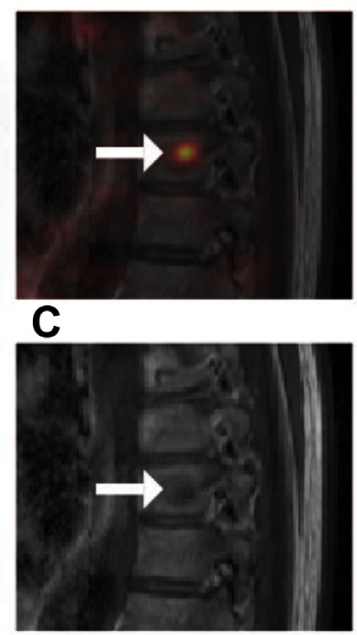

D

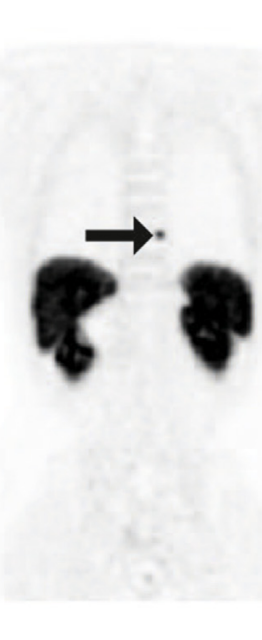

E

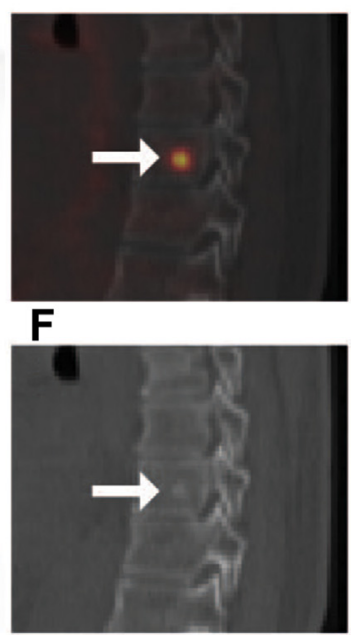

Fig. 3. PSMA-1007 PET/MR $(A-C)$ and PET/CT $(D-F)$ images of a patient with suspected bone metastases (arrows). Both PET from PET/MR imaging $(A)$ and PET from PET/CT $(D)$ show focal uptake in the spine on the coronal images. Both PET image fusion with the sagittal T1w turbo spin echo (TSE) image $(B)$ and PET image fusion with the sagittal low-dose CT $(E)$ provide anatomic reference in the bony tissue. However, the morphologic correlate to the suspected bone lesion may be more clearly visualized on the sagittal T1w TSE image than on the sagittal low-dose CT image $(F)$. 
Table 1

The PET/magnetic resonance imaging scan protocol and corresponding scan times (minutes) used on the 3-T Biograph MMR scanner (Siemens Healthineers, Erlangen, Germany) in our institution

\begin{tabular}{ll} 
MR Imaging Sequence & $\begin{array}{l}\text { Simultaneous } \\
\text { PET }\end{array}$ \\
\hline Localizers whole body (2:45) & No \\
\hline 4 bed positions & Yes $(6: 00)$ \\
MRAC (0:15) & \\
T1w VIBE COR (0:15) & \\
T2w HASTE TRA (1:15) & \\
DW EPI TRA (2:28) & \\
T1w TSE SAG (0:58) & No \\
\hline Localizer pelvis (0:57) & Yes (10:00) \\
\hline 1 bed position & \\
MRAC (0:15) & No \\
T2W SPACE (08:15) & No \\
\hline DW EPI TRA (3:33) & \\
\hline DCE T1w VIBE TRA (3:29)
\end{tabular}

In patients who have undergone prostatectomy, typical high-resolution T2w turbo spin echo imaging of the prostate bed in 3 orthogonal planes is deemed unnecessary when high-resolution $\left(0.5 \times 0.5 \times 1 \mathrm{~mm}^{3}\right)$ T2w SPACE images are also available.

Abbreviations: COR, coronal; DW EPI, Diffusionweighted echo planar imaging; HASTE, half-Fourier acquired single-shot turbo spin echo; MRAC, MR imagingbased attenuation correction; SAG, sagittal; SPACE, Sampling Perfection with Application optimized Contrasts using different flip angle Evolution; T1w, T1 weighted; TRA, transverse; TSE, turbo spin echo; VIBE, volumetric interpolated breath-hold examination.

have suggested a PSMA PET/CT-based workflow, ${ }^{47,48}$ but this approach would still suffer from the inherent limitations of CT. For example, Habl and colleagues ${ }^{49}$ reported that additional planning MR imaging needed to be performed when PSMA PET/CT suggested local recurrence, because morphologic correlates were usually lacking in the CT scan. In contrast, a PSMA PET/MR imaging-based RT planning workflow could provide all the information needed for delineation of the target volumes and organs at risk.

\section{IMPACT OF PROSTATE-SPECIFIC MEMBRANE ANTIGEN PET ON PATIENT MANAGEMENT}

According to international guidelines, ${ }^{5}$ patients with BCR after RP should be treated with $\mathrm{SRT}$ when PSA levels are less than $0.5 \mathrm{ng} / \mathrm{mL}$, presuming the site of recurrence is locally in the prostate bed. It has been shown that the therapeutic effect of SRT reduces with increasing PSA levels, ${ }^{12,13}$ which probably reflects an increased likelihood of occult metastatic disease outside the radiation field. However, even in the low-PSA group, more than $40 \%$ of patients treated with sRT do not achieve long-term PSA response. ${ }^{50}$ New insights into the pattern of prostate cancer recurrence came to light with the introduction of PSMA PET imaging, among other things convincingly showing that low PSA values do not guarantee absence of metastatic disease. ${ }^{29,47}$ For example, one study by Meredith and colleagues ${ }^{29}$ reported suspected local recurrence, lymph node metastases, and bone metastases in $2 \%, 7 \%$, and $2 \%$ of patients with PSA level less than $0.2 \mathrm{ng} / \mathrm{mL}$, respectively, and in $8 \%, 13 \%$, and $6 \%$ of patients with PSA 0.2 to less than $0.5 \mathrm{ng} / \mathrm{mL}$. This pattern of recurrence at low PSA values mimics more or less the overall pattern described in the meta-analysis by Perera and colleagues, ${ }^{28}$ albeit with lower detection rates. Consequently, PSMA PET imaging may be the key to improving the efficacy of sRT, by unblinding the patient selection and treatment planning process, and a substantial impact on patient management may be expected.

A recent meta-analysis by Han and colleagues ${ }^{51}$ showed that PSMA PET imaging changed treatment decisions in about half of all patients with prostate cancer. They pooled the results obtained from studies with a mix of patients with prostate cancer, including those with primary prostate cancer, BCR after RP, and BCR after RT, and found an overall proportion of (intended or implemented) change in management of $54 \%$. PET positivity was the only variable significantly associated with a change in management. In patients with BCR after initial treatment (RP, RT, or both), the availability of PSMA PET led to increased use of curative treatment options at the expense of palliative systemic treatment. The proportion of patients assigned to radiotherapy increased from $56 \%$ before PSMA PET to $62 \%$ after PSMA PET, surgery increased from $1 \%$ to $7 \%$, focal treatment from $1 \%$ to $2 \%$, and multimodal treatment from $2 \%$ to $6 \%$. In contrast, the proportion of patients assigned to systemic treatment decreased from $26 \%$ to $12 \%$ because of PSMA PET, and those without treatment decision decreased from 14\% to $11 \%$. Conventional SRT to the prostate bed was the RT methodology of choice in $95 \%$ of the patients before PSMA PET imaging. However, after PSMA PET, $24 \%$ of patients received SRT with increased dose to the sites of suspected recurrence and/or an enlarged target volume, including the sites of recurrence, and $20 \%$ received stereotactic body $\mathrm{RT}$ to single metastases or oligometastases. There was a tendency toward a greater proportion of change in patient management in studies that included relapsing 
patients with higher PSA levels $(43 \%$ for PSA $<1 \mathrm{ng} / \mathrm{mL}, 54 \%$ for PSA 1 to $<2 \mathrm{ng} / \mathrm{mL}$, and $69 \%$ for PSA $>2 \mathrm{ng} / \mathrm{mL}$ ).

For this work, 10 studies that specifically reported the overall change in management in patients with BCR after RP were identified (Table 2). Major changes in treatment management were reported in $19 \%$ to $77 \%$ of patients, with a $43 \%$ median proportion of change. This finding agrees well with the pooled proportion of change for the lowest PSA level group $(<1 \mathrm{ng} / \mathrm{mL})$ reported by Han and colleagues, ${ }^{51}$ which is based on patients initially treated with RP $( \pm R T)$ only. ${ }^{49,52,53}$ Seven out of 10 studies explicitly reported the changes in treatment decisions. ${ }^{47-49,52-55}$ Van Leeuwen and colleagues ${ }^{53}$ investigated the impact of PMSA PET in men who were diagnosed with BCR after RP and were being considered for SRT to the prostate bed. PSMA PET changed the treatment in 20 out of $70(29 \%)$ patients, including enlarging the RT treatment volume to the pelvic nodes in 5 out of $20(25 \%)$ patients, changing to surgical salvage lymph node dissection in 1 out of $20(5 \%)$ patients, $\mathrm{RT}$ to the pelvic lymph nodes plus ADT in 6 out of 20 (30\%) patients, stereotactic RT of a solitary pelvic lymph node in 4 out of $20(20 \%)$ patients, stereotactic $\mathrm{RT}$ of a lesion outside the pelvis with or without ADT in 3 out of $20(15 \%)$ patients, and $\mathrm{RT}$ to the prostatic fossa plus stereotactic radiotherapy for an extrapelvic lesion in 1 out of 20 (5\%) patients. Bluemel and colleagues ${ }^{54}$ investigated the impact of PSMA PET on patient management in men with persistent PSA or BCR after RP and who were candidates for SRT to the prostate bed. Treatment recommendations changed in 19 out of 42 (42\%) patients because of findings on PSMA PET. These changes included dose escalation to local recurrence in 6 out of 19 patients (32\%); extension of the RT field to a suspicious rectal lesion in 2 out of 19 (11\%) patients; extension of the RT field to pelvic and/or retroperitoneal lymph node metastases, including dose escalation for involved lymph nodes in 8 out of 19 (42\%) patients; extension of the RT field to lymph node metastases and a single bone metastasis in 1 out of $19(5 \%)$ patients; and change to systemic treatment with ADT in 2 out of $19(11 \%)$ patients with multiple distant bone metastases and/or multiple lymph node metastases. Habl and colleagues $^{49}$ investigated the impact of PSMA PET on SRT treatment planning in 100 patients with BCR after RP, with or without prior RT. Only patients who received SRT after PSMA PET imaging were considered, thus, for example, excluding patients with multiple metastases who received ADT or other therapies. The conventional RT treatment plan (prostate bed) was changed in 59 out of 100 (59\%) patients. These plans included RT plus simultaneous integrated boost (SIB) to the prostate bed in 19 out of 59 (32\%) patients, RT plus SIB to the pelvic lymph nodes in 22 out of $59(37 \%)$ patients, RT plus SIB to extrapelvic lymph nodes in 15 out of $59(25 \%)$ patients, stereotactic $\mathrm{RT}$ to a single lymph node metastasis in 1 out of $59(2 \%)$ patients, and stereotactic RT bone metastases in 10 out of 59 (17\%) patients. Zschaek and colleagues ${ }^{55}$ investigated the impact of PSMA PET on treatment management in highrisk patients presenting with BCR after RP and who were candidates for sRT. All 22 patients were originally scheduled for SRT of the prostate bed. PSMA PET led to treatment modifications in 17 out of $22(77 \%)$ patients, including an additional

Table 2

Overview of studies that report the impact of prostate-specific membrane antigen PET on treatment decisions, specifically for patients with prostate cancer with biochemical recurrence after radical prostatectomy

\begin{tabular}{llllll} 
Study by First Author & N & $\begin{array}{l}\text { Average } \\
\text { Age }(\mathbf{y})\end{array}$ & $\begin{array}{l}\text { Average } \\
\text { PSA (ng/mL) }\end{array}$ & $\begin{array}{l}\text { Detection } \\
\text { Rate (\%) }\end{array}$ & $\begin{array}{l}\text { Proportion of Change in } \\
\text { Management (\%) }\end{array}$ \\
\hline Sterzing et al, ${ }^{56} 2016$ & 42 & 70 & 2.8 & 74 & 61 \\
\hline Van Leeuwen et al, ${ }^{53} 2016$ & 70 & 67 & 0.2 & 54 & 29 \\
\hline Bluemel et al, $^{54} 2016$ & 46 & 69 & 0.7 & 53 & 42 \\
\hline Habl et al, ${ }^{49} 2017$ & 100 & 64 & 0.7 & 71 & 59 \\
\hline Hope et al, ${ }^{57} 2017$ & 43 & 69 & 2.7 & - & 33 \\
\hline Afaq et al, ${ }^{58} 2018$ & 68 & - & - & - & 34 \\
\hline Zschaeck et al, $^{55} 2017$ & 22 & 65 & 6.1 & - & 77 \\
\hline Grubmüller et al, ${ }^{52} 2018$ & 117 & 74 & 1.0 & 86 & 43 \\
\hline Henkenberens et al, ${ }^{48} 2018$ & 39 & 66 & 1.2 & 85 & 59 \\
\hline Calais et al, ${ }^{47} 2018$ & 270 & 68 & 0.4 & 49 & 19 \\
\hline
\end{tabular}


dose boost to local recurrence in 6 out of 17 (35\%) patients, extension of the RT field plus an additional dose boost to the affected lymph nodes in 10 out of 17 (59\%) patients, and stereotactic RT of bone metastases in 4 out of 17 (24\%) patients. In 1 out of $17(6 \%)$ patients, RT was omitted because of extensive metastatic disease. Grubmuller and colleagues ${ }^{52}$ found that PSMA PET changed the clinical decision-making in 50 out of 117 (42\%) patients with BCR after RP, with or without prior RT. RT to the prostate bed was changed to wait and see in 1 out of $50(2 \%)$ patients, to salvage surgery in 1 out of $50(2 \%)$ patients, and to metastases-directed RT in 1 out of $50(2 \%)$ patients. ADT was changed to wait and see in 1 out of $50(2 \%)$ patients, to salvage surgery in 10 out of $50(20 \%)$ patients, to metastasesdirected RT in 13 out of $50(26 \%)$ patients, and to multimodal treatment in 5 out of $50(10 \%)$ patients. Wait and see was changed to salvage surgery in 2 out of 50 (4\%) patients and to metastases-directed RT in 16 out of 50 (32\%) patients. Henkenberens and colleagues ${ }^{48}$ also investigated the impact of PSMA PET on the management of relapsing patients with a high risk of metastatic disease. Treatment was changed from conventional SRT to the prostate bed to individualized treatment concepts in 23 out of $39(59 \%)$ patients, including metastasesdirected RT with or without ADT in 19 out of 23 (83\%) patients, ADT alone in 2 out of $23(9 \%)$ patients, chemotherapy alone in 1 out of $23(4 \%)$ patients, and ADT plus chemotherapy in 1 out of 23 (4\%) patients. In addition, Calais and colleagues ${ }^{47}$ investigated the potential impact of PSMA PET on the planning of SRT in patients with PSA levels less than $1 \mathrm{ng} / \mathrm{mL}$. Major impact was found in 52 out of $270(19 \%)$ patients, including extension of the pelvic target volume in 19 out of 52 (37\%) patients, extension of the target volume to the para-aortic lymph nodes in 5 out of $52(10 \%)$ patients, and metastases-directed stereotactic body RT in 22 out of $52(42 \%)$ patients. In 6 out of $52(12 \%)$ patients, RT would have been avoided because of advanced metastatic disease. In addition, 80 out of $270(30 \%)$ patients could have received focal dose escalation to lesions within the original target volume, which was considered as minor impact by the investigators.

In summary, these initial studies indicate that PSMA PET can have substantial impact on the planning of SRT in patients with prostate cancer with BCR after RP. Some patients received palliative systemic treatment because PSMA PET indicated that SRT would be futile because of the presence of advanced metastatic disease. In other patients, long-term ADT was modified to locoregional SRT, thereby potentially curing the disease and reducing, or at least delaying, systemic side effects. In a substantial number of patients, the RT plan was adapted to include all sites of recurrence, to boost the dose to suspected lesions, and/or to treat single or oligometastatic disease. In others, PSMA PET revealed that active treatment and not a wait-and-see policy would be the best treatment strategy to avoid progression of existing disease. Importantly, these findings cannot be generalized to all relapsing patients after RP because of limitations with regard to retrospective study design, low numbers of patients, mixed populations, and patient selection bias. Furthermore, long-term follow-up data are currently lacking, so it remains to be seen whether PSMA PET-based treatment planning will lead to improved overall survival. Two prospective randomized controlled trials (ClinicalTrials.gov identifier: NCT03525288, NCT03582774) are currently underway to shed more light on these important questions.

\section{SUMMARY}

$\mathrm{sRT}$ is a potentially curative treatment option for patients with prostate cancer with BCR after RP, especially when PSA levels are still low. Because of a lack of sensitive conventional imaging methods, the planning of sRT is currently being performed in the absence of visual evidence of the site of recurrence. Simultaneous PSMA PET/MR imaging combines excellent soft tissue contrast with a superior sensitivity for cancer, and can help detect recurrence in the prostate bed, lymph nodes, bones, and distant organs. Consequently, PSMA $\mathrm{PET} / \mathrm{MR}$ imaging can play an important role in the selection of patients who may benefit from sRT, and facilitate dose-escalated and metastasesdirected treatment planning. Whether or not PSMA PET/MR imaging-based planning of sRT can improve the long-term outcome in patients with BCR after RP should be answered in prospective randomized controlled trials.

\section{REFERENCES}

1. Siegel RL, Miller KD, Jemal A. Cancer statistics, 2017. CA Cancer J Clin 2017;67(1):7-30.

2. Mottet N, Bellmunt J, Bolla M, et al. EAU-ESTROSIOG Guidelines on prostate cancer. Part 1: screening, diagnosis, and local treatment with curative intent. Eur Urol 2017;71(4):618-29.

3. Freedland SJ, Presti JC, Amling CL, et al. Time trends in biochemical recurrence after radical prostatectomy: results of the SEARCH database. Urology 2003; 61(4):736-41. Available at: http://www.ncbi.nlm.nih. 
gov/pubmed/12670557. Accessed February 28, 2019.

4. Ward JF, Blute ML, Slezak J, et al. The Long-term clinical impact of biochemical recurrence of prostate cancer 5 or more years after radical prostatectomy. J Urol 2003;170(5):1872-6.

5. Cornford P, Bellmunt J, Bolla M, et al. EAU-ESTROSIOG guidelines on prostate cancer. Part II: treatment of relapsing, metastatic, and castrationresistant prostate cancer. Eur Urol 2017;71(4): 630-42.

6. Sotoudeh H, Sharma A, Fowler KJ, et al. Clinical application of PET/MRI in oncology. J Magn Reson Imaging 2016;44(2):265-76.

7. Kwon HW, Becker A-K, Goo JM, et al. FDG wholebody PET/MRI in oncology: a systematic review. Nucl Med Mol Imaging 2017;51(1):22-31.

8. Barbosa F, Queiroz M, Nunes R, et al. Clinical perspectives of PSMA PET/MRI for prostate cancer. Clinics 2018;73(Suppl 1).

9. Thompson IM, Valicenti RK, Albertsen P, et al. Adjuvant and salvage radiotherapy after prostatectomy: AUA/ASTRO Guideline. J Urol 2013;190(2):441-9.

10. Fossati N, Karnes RJ, Boorjian SA, et al. Long-term impact of adjuvant versus early salvage radiation therapy in PT3NO prostate cancer patients treated with radical prostatectomy: results from a multiinstitutional series. Eur Urol 2017;71(6):886-93.

11. Stephenson AJ, Scardino PT, Kattan MW, et al. Predicting the outcome of salvage radiation therapy for recurrent prostate cancer after radical prostatectomy. J Clin Oncol 2007;25(15):2035-41.

12. Pfister D, Bolla M, Briganti $A$, et al. Early salvage radiotherapy following radical prostatectomy. Eur Urol 2014;65(6):1034-43.

13. Tendulkar RD, Agrawal S, Gao T, et al. Contemporary update of a multi-institutional predictive nomogram for salvage radiotherapy after radical prostatectomy. J Clin Oncol 2016;34(30):3648-54.

14. Shipley WU, Seiferheld W, Lukka HR, et al. Radiation with or without antiandrogen therapy in recurrent prostate cancer. N Engl J Med 2017;376(5):417-28.

15. Carrie C, Hasbini A, de Laroche G, et al. Salvage radiotherapy with or without short-term hormone therapy for rising prostate-specific antigen concentration after radical prostatectomy (GETUG-AFU 16): a randomised, multicentre, open-label phase 3 trial. Lancet Oncol 2016;17(6):747-56.

16. Pollack A, Karrison TG, Balogh AG, et al. Short term androgen deprivation therapy without or with pelvic lymph node treatment added to prostate bed only salvage radiotherapy: the NRG oncology/RTOG 0534 SPPORT trial. Int J Radiat Oncol 2018;102(5):1605.

17. Kane CJ, Amling CL, Johnstone PA, et al. Limited value of bone scintigraphy and computed tomography in assessing biochemical failure after radical prostatectomy. Urology 2003;61(3):607-11.
18. Gaur S, Turkbey B. Prostate MR imaging for posttreatment evaluation and recurrence. Radiol Clin North Am 2018;56(2):263-75.

19. Hövels AM, Heesakkers RAM, Adang EM, et al. The diagnostic accuracy of CT and MRI in the staging of pelvic lymph nodes in patients with prostate cancer: a meta-analysis. Clin Radiol 2008;63(4):387-95.

20. Krause BJ, Souvatzoglou M, Tuncel M, et al. The detection rate of $[11 \mathrm{C}]$ Choline-PET/CT depends on the serum PSA-value in patients with biochemical recurrence of prostate cancer. Eur J Nucl Med Mol Imaging 2008;35(1):18-23.

21. Castellucci P, Ceci F, Graziani T, et al. Early biochemical relapse after radical prostatectomy: which prostate cancer patients may benefit from a restaging 11C-Choline PET/CT scan before salvage radiation therapy? J Nucl Med 2014;55(9):1424-9.

22. Giovacchini G, Picchio M, Briganti A, et al. [ ${ }^{11} \mathrm{C}$ ] Choline positron emission tomography/computerized tomography to restage prostate cancer cases with biochemical failure after radical prostatectomy and no disease evidence on conventional imaging. J Urol 2010;184(3):938-43. https://doi.org/10.1016/ j.juro.2010.04.084.

23. Nanni C, Zanoni L, Pultrone C, et al. 18F-FACBC (anti1-amino-3-18F-fluorocyclobutane-1-carboxylic acid) versus $11 \mathrm{C}$-choline PET/CT in prostate cancer relapse: results of a prospective trial. Eur $\mathrm{J} \mathrm{Nucl}$ Med Mol Imaging 2016;43(9):1601-10.

24. Morigi JJ, Stricker PD, van Leeuwen PJ, et al. Prospective comparison of 18F-Fluoromethylcholine versus 68Ga-PSMA PET/CT in prostate cancer patients who have rising PSA after curative treatment and are being considered for targeted therapy. J Nucl Med 2015;56(8):1185-90.

25. Afshar-Oromieh A, Zechmann CM, Malcher A, et al. Comparison of PET imaging with a 68Ga-labelled PSMA ligand and 18F-choline-based PET/CT for the diagnosis of recurrent prostate cancer. Eur J Nucl Med Mol Imaging 2014;41(1):11-20.

26. Schwenck J, Rempp H, Reischl G, et al. Comparison of 68Ga-labelled PSMA-11 and 11C-choline in the detection of prostate cancer metastases by PET/ CT. Eur J Nucl Med Mol Imaging 2017;44(1):92-101.

27. Evans JD, Jethwa KR, Ost P, et al. Prostate cancerspecific PET radiotracers: a review on the clinical utility in recurrent disease. Pract Radiat Oncol 2018;8(1): 28-39.

28. Perera M, Papa N, Roberts $M$, et al. Gallium-68 prostate-specific membrane antigen positron emission tomography in advanced prostate cancer-updated diagnostic utility, sensitivity, specificity, and distribution of prostate-specific membrane antigenavid lesions: a systematic review and meta-analysis. Eur Urol 2019. https://doi.org/10.1016/J.EURURO. 2019.01.049. 
29. Meredith G, Wong D, Yaxley J, et al. The use of 68 Ga-PSMA PET CT in men with biochemical recurrence after definitive treatment of acinar prostate cancer. BJU Int 2016;118:49-55.

30. Perera M, Papa N, Christidis D, et al. Sensitivity, specificity, and predictors of positive 68 Ga-prostatespecific membrane antigen positron emission tomography in advanced prostate cancer: a systematic review and meta-analysis. Eur Urol 2016;70(6):926-37.

31. Panebianco V, Barchetti F, Sciarra A, et al. Prostate cancer recurrence after radical prostatectomy: the role of 3-T diffusion imaging in multi-parametric magnetic resonance imaging. Eur Radiol 2013; 23(6): 1745-52.

32. Freitag MT, Radtke JP, Afshar-Oromieh A, et al. Local recurrence of prostate cancer after radical prostatectomy is at risk to be missed in 68GaPSMA-11-PET of PET/CT and PET/MRI: comparison with mpMRl integrated in simultaneous PET/MRI. Eur J Nucl Med Mol Imaging 2017;44(5):776-87.

33. Lütje S, Cohnen J, Gomez B, et al. Integrated 68GaHBED-CC-PSMAPET/MRI in patients with suspected recurrent prostate cancer. Nuklearmedizin 2017; 56(03):73-81.

34. Alonso O, dos Santos G, García Fontes M, et al. 68Ga-PSMA and 11C-Choline comparison using a tri-modality PET/CT-MRI (3.0 T) system with a dedicated shuttle. Eur J Hybrid Imaging 2018;2(1):9.

35. Lake ST, Greene KL, Westphalen AC, et al. Optimal MRI sequences for 68Ga-PSMA-11 PET/MRI in evaluation of biochemically recurrent prostate cancer. EJNMMI Res 2017;7(1):77.

36. Dyrberg E, Hendel HW, Huynh THV, et al. 68GaPSMA-PET/CT in comparison with 18F-fluoridePET/CT and whole-body MRI for the detection of bone metastases in patients with prostate cancer: a prospective diagnostic accuracy study. Eur Radiol 2019;29(3):1221-30.

37. Zacho HD, Nielsen JB, Afshar-Oromieh A, et al. Prospective comparison of 68Ga-PSMA PET/CT, 18F-sodium fluoride PET/CT and diffusion weighted-MRI at for the detection of bone metastases in biochemically recurrent prostate cancer. Eur J Nucl Med Mol Imaging 2018;45(11):1884-97.

38. Freitag MT, Radtke JP, Hadaschik BA, et al. Comparison of hybrid 68Ga-PSMA PET/MRI and 68GaPSMA PET/CT in the evaluation of lymph node and bone metastases of prostate cancer. Eur J Nucl Med Mol Imaging 2016;43(1):70-83.

39. Afshar-Oromieh A, Haberkorn U, Schlemmer HP, et al. Comparison of PET/CT and PET/MRI hybrid systems using a 68Ga-labelled PSMA ligand for the diagnosis of recurrent prostate cancer: initial experience. Eur J Nucl Med Mol Imaging 2014; 41(5):887-97.

40. Ringheim A, Campos Neto GC, Martins KM, et al. Reproducibility of standardized uptake values of same-day randomized 68Ga-PSMA-11 PET/CT and PET/MR scans in recurrent prostate cancer patients. Ann Nucl Med 2018;32(8):523-31.

41. Giesel FL, Will L, Lawal I, et al. Intraindividual comparison of ${ }^{18}$ F-PSMA-1007 and 18 F-DCFPyL $\mathrm{PET} / \mathrm{CT}$ in the prospective evaluation of patients with newly diagnosed prostate carcinoma: a pilot study. J Nucl Med 2018;59(7):1076-80.

42. Heußer T, Mann P, Rank CM, et al. Investigation of the halo-artifact in 68Ga-PSMA-11-PET/MRI. PLoS One 2017;12(8):e0183329. Thierry B, ed.

43. Freitag MT, Kesch C, Cardinale J, et al. Simultaneous whole-body 18F-PSMA-1007-PET/MRI with integrated high-resolution multiparametric imaging of the prostatic fossa for comprehensive oncological staging of patients with prostate cancer: a pilot study. Eur J Nucl Med Mol Imaging 2018;45(3): 340-7.

44. Lee E, Park W, Ahn SH, et al. Interobserver variation in target volume for salvage radiotherapy in recurrent prostate cancer patients after radical prostatectomy using CT versus combined CT and MRI: a multicenter study (KROG 13-11). Radiat Oncol J 2018;36(1):11-6.

45. Villeirs GM, Van Vaerenbergh $K$, Vakaet $L$, et al. Interobserver delineation variation using CT versus combined $\mathrm{CT}+\mathrm{MRI}$ in intensity-modulated radiotherapy for prostate cancer. Strahlenther Onkol 2005;181(7):424-30.

46. Kerkmeijer LGW, Maspero M, Meijer GJ, et al. Magnetic resonance imaging only workflow for radiotherapy simulation and planning in prostate cancer. Clin Oncol 2018;30(11):692-701.

47. Calais J, Czernin J, Cao M, et al. 68Ga-PSMA-11 PET/CT mapping of prostate cancer biochemical recurrence after radical prostatectomy in 270 patients with a PSA level of less than $1.0 \mathrm{ng} / \mathrm{mL}$ : impact on salvage radiotherapy planning. J Nucl Med 2018; 59(2):230-7.

48. Henkenberens C, Derlin T, Bengel FM, et al. Patterns of relapse as determined by 68Ga-PSMA ligand PET/CT after radical prostatectomy. Strahlenther Onkol 2018;194(4):303-10.

49. Habl G, Sauter K, Schiller K, et al. ${ }^{68}$ Ga-PSMA-PET for radiation treatment planning in prostate cancer recurrences after surgery: individualized medicine or new standard in salvage treatment. Prostate 2017;77(8):920-7.

50. Stish BJ, Pisansky TM, Harmsen WS, et al. Improved metastasis-free and survival outcomes with early salvage radiotherapy in men with detectable prostate-specific antigen after prostatectomy for prostate cancer. J Clin Oncol 2016;34(32):3864-71.

51. Han S, Woo S, Kim YJ, et al. Impact of 68Ga-PSMA PET on the management of patients with prostate cancer: a systematic review and meta-analysis. Eur Urol 2018;74(2):179-90. 
52. Grubmüller B, Baltzer P, D'Andrea D, et al. 68GaPSMA 11 ligand PET imaging in patients with biochemical recurrence after radical prostatectomy - diagnostic performance and impact on therapeutic decision-making. Eur J Nucl Med Mol Imaging 2018;45(2):235-42.

53. van Leeuwen PJ, Stricker P, Hruby G, et al. ${ }^{68}$ GaPSMA has a high detection rate of prostate cancer recurrence outside the prostatic fossa in patients being considered for salvage radiation treatment. BJU Int 2016;117(5):732-9.

54. Bluemel C, Linke F, Herrmann K, et al. Impact of 68Ga-PSMA PET/CT on salvage radiotherapy planning in patients with prostate cancer and persisting PSA values or biochemical relapse after prostatectomy. EJNMMI Res 2016;6(1):78.
55. Zschaeck S, Wust P, Beck M, et al. Intermediateterm outcome after PSMA-PET guided high-dose radiotherapy of recurrent high-risk prostate cancer patients. Radiat Oncol 2017;12(1):140.

56. Sterzing F, Kratochwil C, Fiedler H, et al. 68Ga-PSMA11PET/CT: a new technique with high potential for the radiotherapeutic management of prostate cancer patients. Eur J Nucl Med Mol Imaging 2016;43(1):34-41.

57. Hope TA, Aggarwal R, Chee B, et al. Impact of 68Ga-PSMA-11 PET on management in patients with biochemically recurrent prostate cancer. J Nucl Med 2017;58(12):1956-61.

58. Afaq A, Alahmed S, Chen S-H, et al. Impact of 68Ga-prostate-specific membrane antigen PET/CT on prostate cancer management. J Nucl Med 2018;59(1):89-92. 